\title{
Impinging jets array: an experimental investigation and numerical modeling
}

\author{
Nilesh Dhondoo ${ }^{1, *}$, Ștefan-Mugur Simionescu ${ }^{1}$, and Corneliu Bălan ${ }^{1}$ \\ ${ }^{1}$ REOROM Group, Department of Hydraulics, Hydraulic Machinery and Environmental Engineering, Faculty of Power Engineering, \\ University "Politehnica" of Bucharest, Romania
}

\begin{abstract}
This paper reports on the measurements of wall shear stress and static pressure along a smooth static wall upon which jet impingement occurs. The effect of a single circular jet, respectively an array of jets is studied using a high speed/resolution camera. The areas of interest are the stagnation region and the wall jet region, where the jet is deflected from axial to radial direction. The effect of increasing the distance between the inlets is also investigated. The results are obtained by performing direct flow experimental visualizations and CFD numerical simulations, using the Reynolds averaged Navier-Stokes (RANS) approach with the commercial software ANSYS Fluent. The findings suggest that the smaller the nozzle-towall distance is, the higher the pressure peak. The wall shear stress has a bimodal distribution; at stagnation point, the wall shear stress is 0 . An increase in the number of inlets produces the effect of a decrease in the stagnation point pressure. The greater the inter-inlet distance is, the greater the stagnation point pressure (there is less inter-jet mixing, less energy is lost in vortices formed between jets).
\end{abstract}

\section{Introduction}

The study of impinging jet flows (IJF) has been prompted by the late 1950s efforts to design a vehicle capable to withstand high atmospheric entry temperatures - the high heat transfer coefficient of jets impinging on a solid surface make them the ideal solution for this problem. Hrycak et al. (1974) [1] underline that the investigation of the topics connected with the heat transfer for the impinging jets can be traced back to about 1951 for an experimental approach and to 1956 for a combination of analysis and experimentation.

Ever since, IJFs have found applications in various fields: drying in paper industry, cooling and heating in food industry, annealing metallurgy and cooling of heated components in gas turbine engines, computers and electronic instruments (Sastry et. al, 2015, [2]). The diversification of IJF applications can be attributed to their ability to control the heat transfer by changing parameters like nozzle diameter, the nozzle-to-wall distance, the number of nozzles, the surface geometry, etc.

With the arrival of Computational Fluid Dynamics (CFD) software, the governing equations of the flow could be solved numerically by dividing the fluid volume into grids of discrete elements and finding the velocity and pressure in each node.

When a jet fluid exiting a nozzle encounters a flat plate, its behaviour changes - the impingement wall influences the flow parameters (like pressure and

velocity). Still, many studies suggest that this influence is felt only in the vicinity of the wall: for example, Tani and Komatsu [3] have shown that for a circular fluid jet, the effect of the impingement wall on the jet is undetectable at a distance over 1 nozzle diameter from the wall. Beyond this distance, the fluid behaves as a free jet.

This article looks into the flow dynamics of one, respectively several circular air jets impinging on a smooth flat plate. An experimental investigation is carried out, and the results are discussed. A quantitative analysis is performed by extracting information from sets of pictures taken during the experiments - at a rate of $400 \mathrm{fps}$. In parallel, a numerical investigation using the ANSYS Fluent code is carried out. The flow domain is split into grid cells, the initial and boundary conditions are set to the software. The mathematical model used to solve the governing equations is RANS (Reynolds averaged Navier - Stokes). The analysis consists in evaluating the behaviour of the wall static pressure $p$ and wall shear stress $\sigma_{w}$ distributions along the different regions of the jet. The independent variables chosen are the flow Reynolds number at the inlet of the nozzle, the nozzle diameter $d$, the inter-nozzle distance $D$ and the nozzle-to-wall distance $z / d$. By setting different values for these parameters, the effects they have on the static pressure and wall shear stress distributions are quantified. A comparison between the experimental and CFD results is performed, in order to assess the validity of the model chosen and the accuracy of the CFD results.

* Corresponding author: nilesh08a@,hotmail.com 


\section{Theoretical aspects}

Impingement Jet Flow (IJF) is a type of flow where the jet exits a nozzle and travels as a free jet until it impinges a flat surface. According to Gautner [4], four flow regions can be typically distinguished in an impinging fluid jet-see Fig. 1:

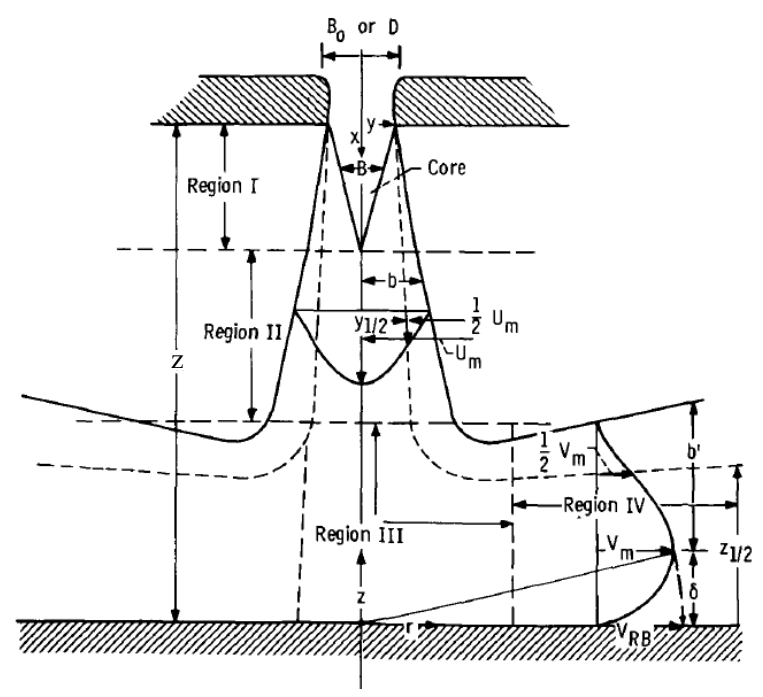

Fig. 1. The typical regions of an impinging jet flow (reproduced after [4]).

* Region I: the region of flow establishment, extending to the apex of potential core region (that is the region in which velocity is constant, being equal to the velocity at the inlet of the nozzle).

* Region II: the region of fully developed flow.

* Region III: the region where the jet impinges on the plate - stagnation region. Here, the jet changes direction from axial to radial.

* Region IV: the wall jet region, where the jet forms a boundary layer on the wall until it detaches from the solid wall.

Shademan et al. (2013) [5] distinguish three regions of flow, based on the $z / d$ value. For $z / d<4$ the potential core zone reaches the impingement plate before it reaches its full length. For $4<z / d<6$ the flow is in a transition zone, as for $z / d>6$, all 4 regions are present. They have used a three-dimensional RANS simulation in order to investigate the effect of $z / d$ on different flow characteristics. Three $z / d$ values have been studied:

a. $z / d=2-$ when the impinging occurs before the potential core is fully developed;

b. $z / d=6-$ when the core has almost reached its full length when hitting the plate;

c. $z / d=18.5-$ a fully developed jet reaches the plate.
Arabnejad et al. (2014) [6] have developed an empirical method for measuring pressure near the wall and an analytical method to measure shear stress applied to oblique circular jets impinging on a smooth wall. Their finding suggest that the flow is quasi-axisymmetric along radial lines with very small shear stresses between radial planes. Moreover, the skin friction coefficient depends only on the inlet Reynolds number.

In their 1996 published paper, Tu and Wood [7] discuss the shape of the wall shear stress distribution in a jet impinging experiment. They varied the nozzle-to-wall distance $z / d$ and the inlet Reynolds number and concluded that the wall shear stress distribution depends on both parameters. They also found that the predicted wall shear stress - pressure relation (from Hiemenz's stagnation flow equation) does not hold; they hypothesize that the discrepancy is due to the high turbulence in the free-stream region of the flow.

Van Hout et al. (2018) [8] studied the vortex structure of a single round nozzle jet impinging on a flat plate. Their results suggest that at low Reynolds number flows (1300) the primary - secondary vortices generated along the wall were "highly coherent". In contrast, for higher Reynolds numbers (6260 and 12354), primary vortices dissipate before the jet hits the wall. Their analysis concludes that "stretching and realignment due to the mean flow always strengthened the vortices while turbulent diffusion mainly weakened them".

\section{Experimental study}

The experiments performed are dealing with air jets formed by one or several circular nozzles and impinging on a flat plate. The equipment used to perform the experiments consists of an impingement plate made of 3 $\mathrm{mm}$ thick plexiglas. The jet is made visible in a lighted plane produced by a continuous planar laser with $532 \mathrm{~nm}$ wavelength. A mirror is used as a laser beam reflector, to increase the laser power in the jet impingement area. The circular nozzles have a diameter of $4.8 \mathrm{~mm}$, and the experiments are performed by using one single jet nozzle, two jet nozzles, respectively three jet nozzles on a line. The jet settling chamber has a volume of $170 \mathrm{dm}^{3}$. The air in the jet is seeded with smoke particles, in order to make it visible in the laser light. The smoke machine used is a Showtec Atmos 1000. The experiment is recorded with a Nikon $1 \mathrm{~J} 5$ camera at the speed of 400 fps and a resolution of $800 \times 296$ pixels. The height of the impingement plate can be adjusted using a vertical regulator, in order to change the nozzle-to-plate distance $z / d$ of the experiment. The Reynolds number is calculated as in equation (1). 


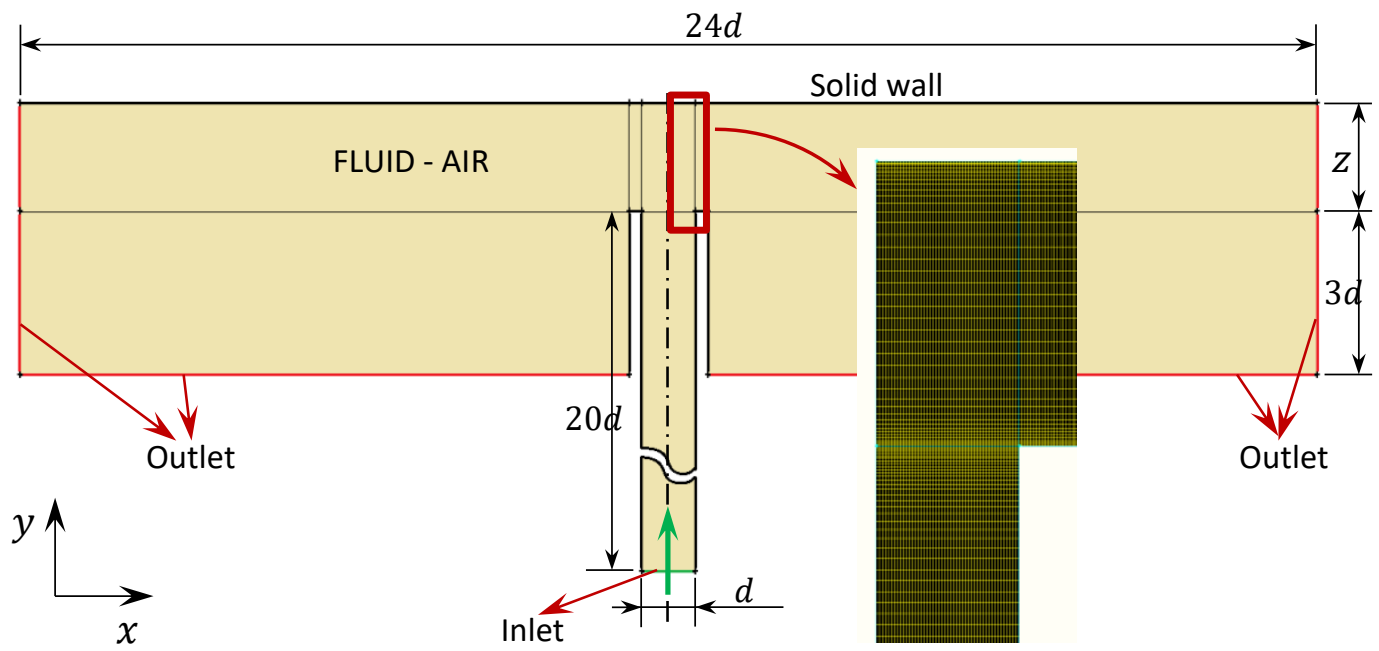

Fig. 2. Sketch of the $2 \mathrm{D}$ numerical domain with boundary conditions and detail of the mesh in the jet impingement region.

$$
R e=\frac{\rho v d}{\eta}
$$

where $\rho$ is the fluid density, $v$ is the average fluid velocity at the nozzle inlet, $d$ is the nozzle diameter and $\eta$ the fluid viscosity.

The average air velocity at the nozzle inlet is assessed by analysing the images captured by the camera, from the moment where the jet exits the nozzle, until the impact.

\section{Numerical study}

A numerical study was performed by using ANSYS Fluent software. A sketch of the flow domain with dimensions is presented in Fig. 2. The geometry and mesh were built by using the software program Gambit. The spatial discretization consists of structured mesh cells. The governing equations have been solved using the RANS model in a laminar regime. The numerical results were validated against the ones extracted from the experimental study, by imposing the same flow domain and boundary conditions. Table 1 shows four cases where we have studied the wall shear stress and pressure distribution as a function of various parameters such as Reynolds number, inter-inlet distance, $z / d$ value and number of inlets.

\section{Results}

Table 2 shows the time evolution of jets exiting 1 inlet, 2 inlets, respectively 3 inlets. In the case of 2 inlets, as from the $309.69 \mathrm{~ms}$ timestamp, the 2 jets start mixing up, resulting to the formation of 2 vortices turning in opposite directions in the inter-nozzle stagnation area. As far as the 3 jets are concerned, they start interacting at the $333.3 \mathrm{~ms}$ timestamp. Rapidly, after $366.6 \mathrm{~ms}$ the inter-jet flow is already turbulent, fact

Table 1. Numerical study cases.

\begin{tabular}{|c|c|c|c|c|c|}
\hline & \multicolumn{4}{|c|}{ Variable parameters } & Constant parameters \\
\hline CASE I & $\begin{array}{l}\text { Nozzle-to-plate } \\
\text { distance, } z / d\end{array}$ & 2 & 4 & 6 & $\begin{array}{c}\mathrm{Re}=1500 \\
D=2 d \\
n=1\end{array}$ \\
\hline CASE II & Number of inlets, $n$ & 1 & 2 & 3 & $\begin{array}{c}R e=1500 \\
z / d=2 \\
D=2 d\end{array}$ \\
\hline CASE III & $\begin{array}{l}\text { Distance between } \\
\text { inlets, } D\end{array}$ & $D=2 d$ & $D=4 d$ & - & $\begin{array}{c}\mathrm{Re}=1500 \\
z / d=4 \\
n=2\end{array}$ \\
\hline CASE IV & $\begin{array}{l}\text { Distance between } \\
\text { inlets, } D\end{array}$ & $D=2 d$ & $D=4 d$ & - & $\begin{array}{c}R e_{1}=300 \\
R e_{2}=600 \\
z / d=2 \\
n=2\end{array}$ \\
\hline
\end{tabular}


Table 2. Time evolution of jets exiting 1 inlet, 2 inlets, respectively 3 inlets.

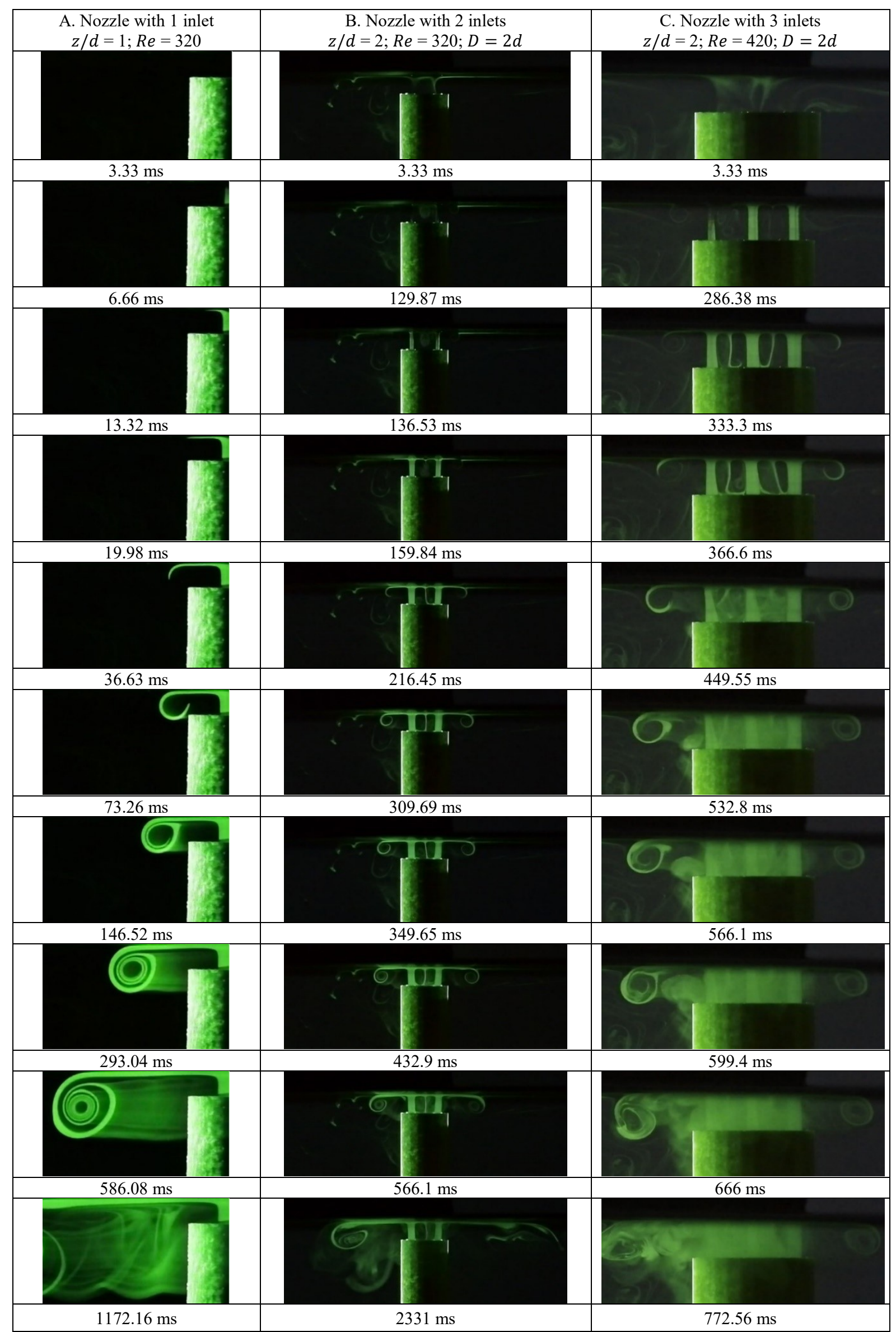


which can be explained by the small $z / d$ value. For the 2 inlets, the evolution of the jets is from the exiting nozzle mouth until they impinge on the flat plate. Pictures are shown at different timestamps, starting with $3.33 \mathrm{~ms}$ to $2331 \mathrm{~ms}$. At $3.33 \mathrm{~ms}$, the jets are still inside the nozzle. The flow is in region I, the potential core. At $136.53 \mathrm{~ms}$, the fully developed flow has already exited the nozzle, being in region II where it starts diffusing in the surrounding fluid; the direction of the flow is axial. At $159.84 \mathrm{~ms}$, the jets impinge on the plate at stagnation point, the flow changes direction becoming radial in region III. At $309.69 \mathrm{~ms}$, the formation of a laminar boundary layer (region IV) attached to the wall can be noticed.

Rapidly the laminar vortex enters a transition phase, followed by a fully turbulent flow with the jet diffusing in the surrounding fluid with the mixing of layers.

\subsection{Case I results}

In Fig. 3 it can be noticed that the static pressure is maximum at $0.015 \mathrm{~mm}$ from the stagnation point, unlike the 1 inlet experiment where the maximum is reached at the stagnation point. The reason for this discrepancy is the fact that the jets from the 2 inlets interact at the stagnation point, creating vortex structures which lead to the drop in the stagnation point pressure.

For smaller $z / d$ values, the maximum pressure is closer to the stagnation point (on either side of it). The greatest static pressure is obtained in the case of the smaller $z / d$ - that is when the plate is closer to the nozzle exit. This can be explained by the fact that in the case of smaller $z / d$ values the jet has lost less of its kinetic energy, hence hitting the wall with a higher pressure.

For $z / d=2$, the maximum static pressure is $2.15 \mathrm{~Pa}$, for $z / d=4$ it is $1.15 \mathrm{~Pa}$ (an almost $50 \%$ decrease), while for the highest $z / d$ value the maximum pressure is 0.9 $\mathrm{Pa}$. In all 3 cases the static pressure decays rapidly. The first static pressure to be 0 is the one in the case of the smallest $z / d$ value.

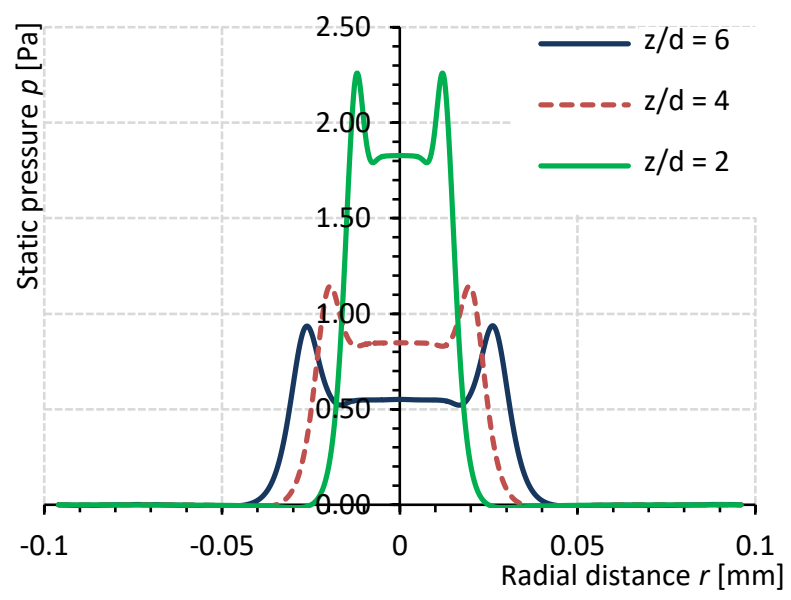

Fig. 3. Static pressure vs radial distance from stagnation point (Case I) at $R e=1500$.
At the stagnation point the wall shear stress is 0 (just like in the case of 1 inlet) - see Fig. 4. The wall shear stress distribution is bimodal (for all the $z / d$ values studied), there is a peak close to the stagnation point and one further away. The wall shear stress peaks decrease rapidly to 0 and peaks higher up once again. The 2 peaks may be due to the interaction between the 2 jets leading to formation of vortices immediately after the stagnation point.

The smaller the $z / d$ value is, the closer the peaks are to the stagnation point similarly (with one exception), for smaller $z / d$ values, the wall shear stress peaks are higher: for $z / d=2$ the 2 peaks are at $0.049 \mathrm{~Pa}$ and 0.13 $\mathrm{Pa}$, for $z / d=4$ the peaks are at $0.022 \mathrm{~Pa}$ and $0.07 \mathrm{~Pa}$. As far as the peaks in the $z / d=6$ case is concerned, the first peak appears at $0.025 \mathrm{~Pa}$ and the second one at $0.06 \mathrm{~Pa}$.

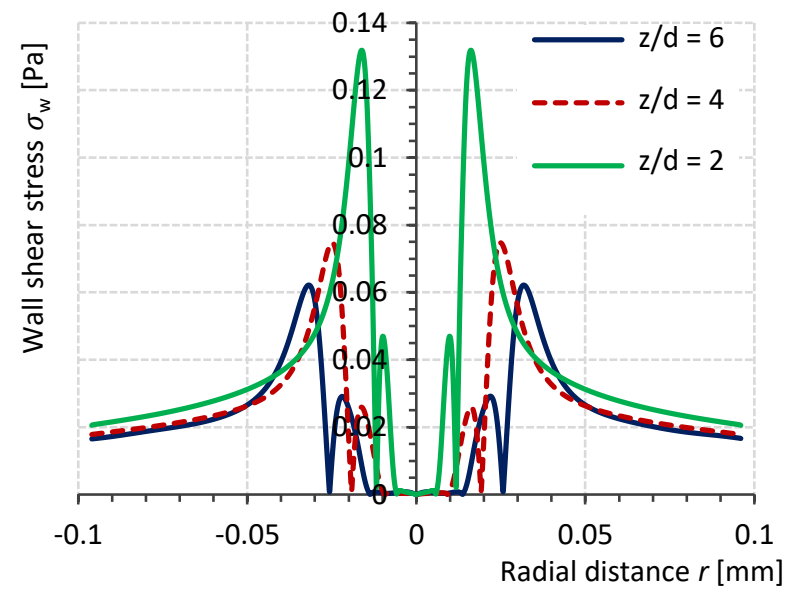

Fig. 4. Wall shear stress vs. radial distance from stagnation point (Case I) at $R e=1500$.

\subsection{Case II results}

According to Fig. 5, when 1 inlet is used, the pressure is maximum (3Pa) at the stagnation point, result which is consistent with previous findings, pressure having a Gaussian distribution. In the case of 2 inlets the stagnation point pressure is $1.9 P a$, while the pressure reaches a maximum value of $2.25 \mathrm{~Pa}$ in the first region of the plate, immediately after the stagnation point. When there are 3 inlets, the pressure is maximum at the stagnation point $(5.5 \mathrm{~Pa})$, but the distribution is not Gaussian, there are 2 local maxima corresponding to the lateral inlets. Very close to the stagnation point, the pressure becomes 0 . The less inlets there are, the faster does the static pressure reach 0 .

The wall shear stress (Fig. 6) is 0 at the stagnation point and it has the greatest value $(0.19 \mathrm{~Pa})$ immediately after the stagnation point in the case of 1 nozzle; the wall shear stress has only one peak of $0.19 \mathrm{~Pa}$ very close to the stagnation point. When 2 nozzles are used, the wall shear stress has the same shape as in Case I, with 2 peaks at $0.042 \mathrm{~Pa}$ and $0.13 \mathrm{~Pa}$. In the inter-inlet area the wall shear stress is 0 . When 3 inlets are used the wall shear stress is 0 at the stagnation point and it has 2 peaks, just as in the case of 2 inlets. The maximum wall shear stress in the case of 1 inlet is 4.5 times higher than the first 
peak in the 2 inlets case and it is 1.4 times higher than the second peak.

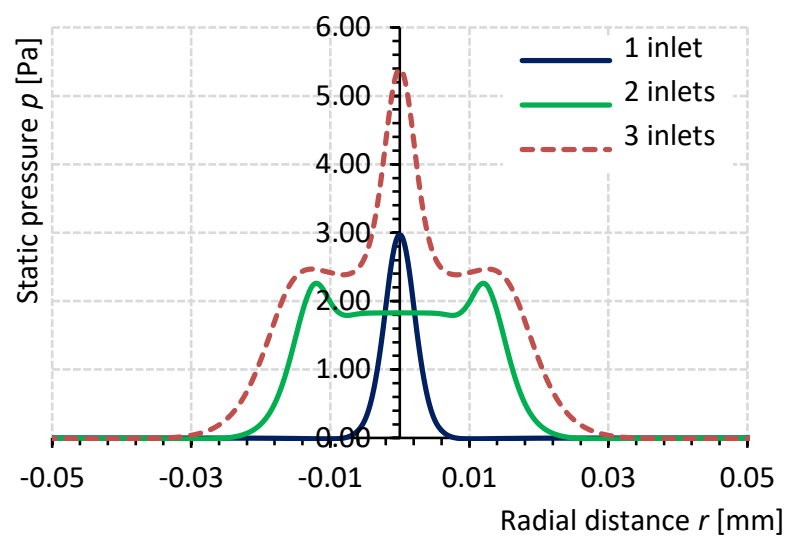

Fig. 5. Static pressure vs radial distance from stagnation point (Case II) at $R e=1500$.

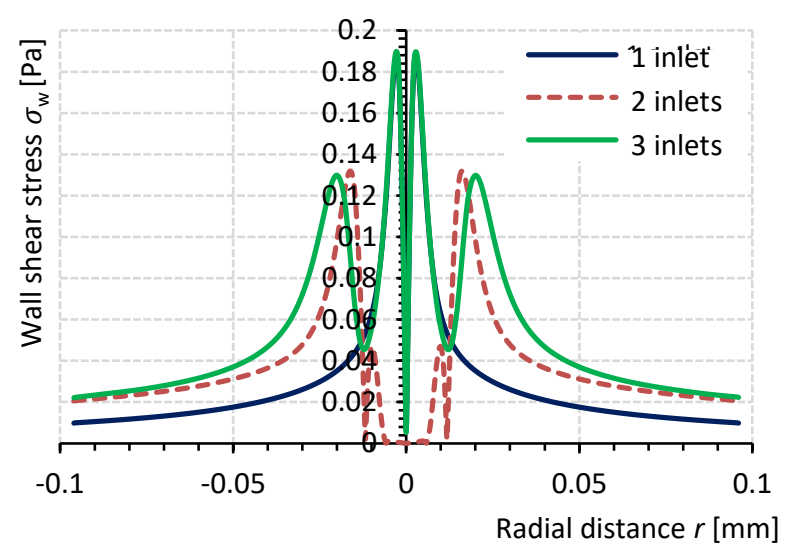

Fig. 6. Wall shear stress vs. radial distance from stagnation point (Case II) at $R e=1500$.

\subsection{Case III results}

A comparison between the pressure distribution for 2 different inter-inlet distances was made. Interestingly, the static pressure is slightly asymmetric when the internozzle distance is $D=4 d$. The smaller the distance between inlets is, the smaller the maximum static pressure is. This can be explained by the fact that inlet proximity implies more inter-jet interaction which in turn leads to the higher pressure.

According to Fig. 7, the maximum pressure of $1.5 \mathrm{~Pa}$ for the $D=4 d$ inter-inlet distance is approximately 1.5 times the maximum pressure in the case of $D=2 d$ interinlet distance.

The same pattern can be noticed (Fig. 8) in the case of the wall shear stress: the distribution is shifted upwards for higher inter-nozzle distance. The first peak in the $D=4 d$ case is twice the peak for the $D=2 d$ case ( $0.5 \mathrm{~Pa}$ versus $0.25 \mathrm{~Pa}$, while the second peaks are very close to each other $(0.9 \mathrm{~Pa}$ versus $0.75 \mathrm{~Pa})$.

The farther away the inlets are, the farther along the plate the wall shear stress peaks. The difference is not significant, though.

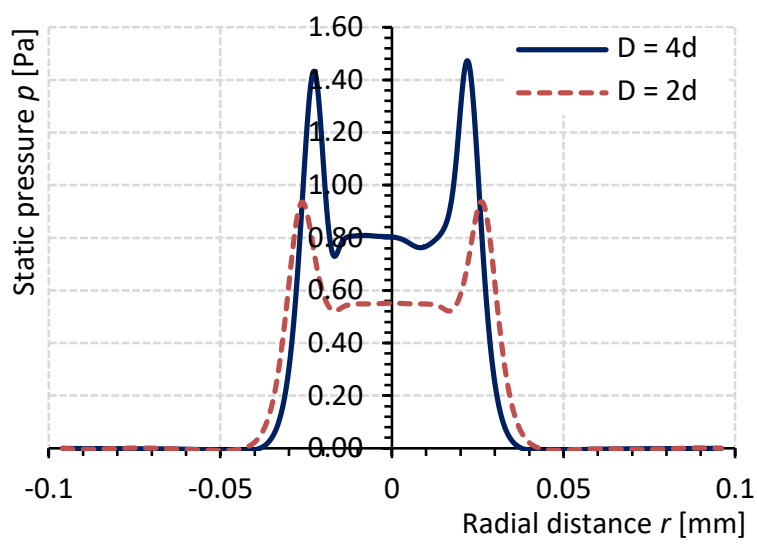

Fig. 7. Static pressure vs radial distance from stagnation point (Case III) at $R e=1500$.

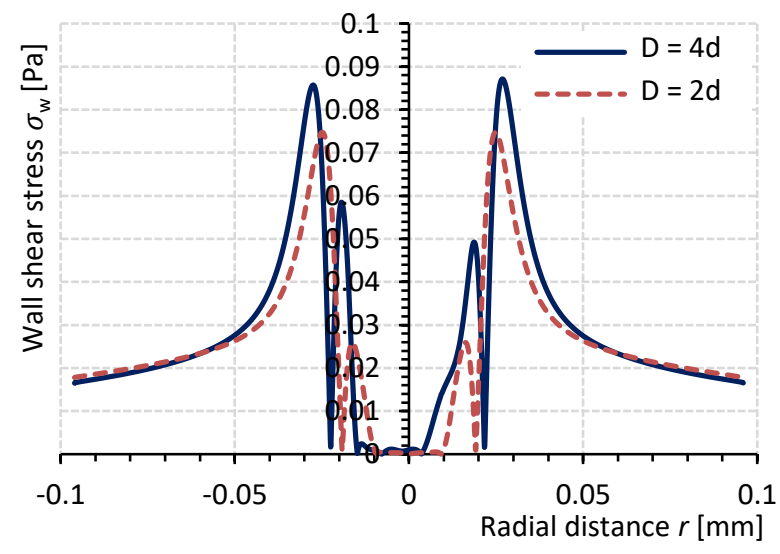

Fig. 8. Wall shear stress vs radial distance from stagnation point (Case III) at $R e=1500$.

\subsection{Case IV results}

When the flows from the 2 nozzles have different Reynolds numbers (one is 300 , the other 600), the experimental results show that the maximum wall shear stress is the same in both cases ( $2 d$ and $4 d$ inter-nozzle distance). However, for nozzles closer to each other, the peaks are reached further along the plate.

The wall shear stress (Fig. 9) distribution is no longer symmetric, unlike the equal Reynolds number flows studied previously. The flow with the smaller Reynolds number has a smaller maximum wall shear stress $(0.07$ $\mathrm{Pa}$ versus $0.21 \mathrm{~Pa}$ for the $D=4 d$ case and $0.06 \mathrm{~Pa}$ versus $0.21 \mathrm{~Pa}$ for the $D=2 d$ case).

The Reynolds number influences the wall shear stress: higher Reynolds number values bring higher wall shear stress. Also, the inter-inlet distance influences the position of the peaks along the plate: the more far apart the inlets are, the closer to the stagnation point the peaks are (unlike the equal Reynolds number case).

The pressure distribution (Fig. 10) is not symmetric, it is flatter for the smaller Reynolds number. This can be explained by fact that the smaller Re number flow hits the plate with less pressure than the higher one does. 


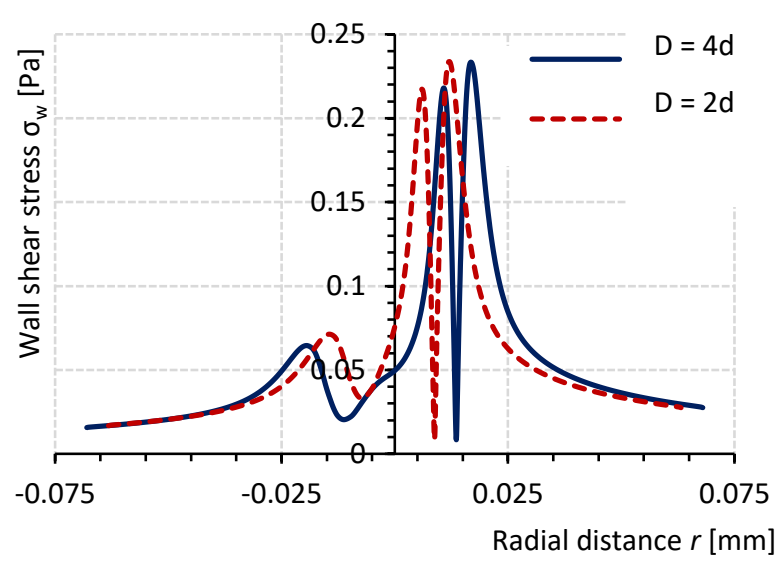

Fig. 9. Wall shear stress vs. radial distance from stagnation point (Case IV) at $R e_{1}=300, R e_{2}=600$.

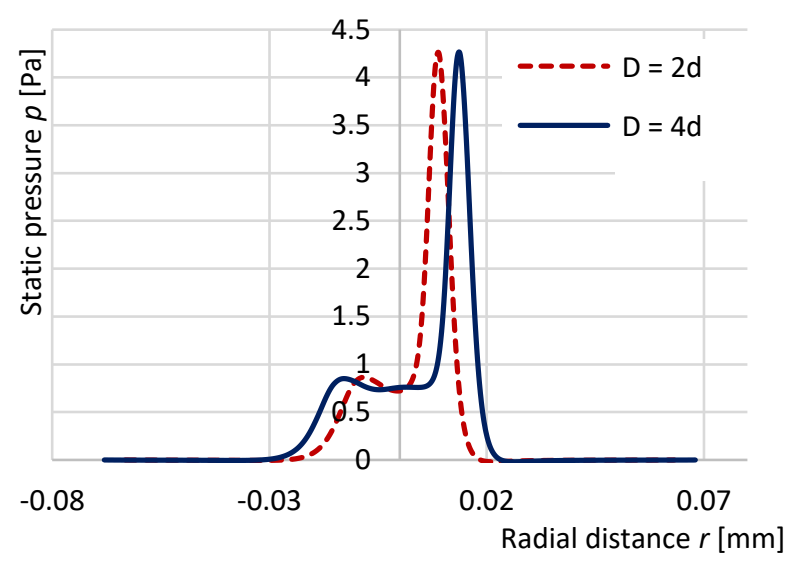

Fig. 10. Static pressure vs radial distance from stagnation point (Case IV) at $R e_{1}=300, R e_{2}=600$.

The inter-inlet distance has no influence on the maximum static pressure value; however, it does influence the point on the plate where this maximum is reached: the higher the distance is, the farther along the plate the pressure peaks.

\section{Conclusions}

When the independent variable is the $z / d$ value (and the other variables: Reynolds number, inter-inlet distance and number of inlets are kept constant), it was found that the nozzle-to-wall distance influences the maximum pressure reached by the jet in the wall region. The smaller the $z / d$ is, the higher the pressure peak. For $z / d$ $=2$, the stagnation point pressure is more than twice the $z / d=4$ stagnation point pressure. The wall shear stress has a bimodal distribution: at stagnation points the wall shear stress is 0 .

The $z / d$ value has a similar influence on the wall shear stress: for smaller $z / d$ values, the wall shear stress peaks are higher. In the inter-jet area the wall shear stress is 0 . When the Reynolds number, the $z / d$ value and the inter-nozzle distance are kept constant but the number of inlets is varied (1, 2 or 3 inlets), an increase in the number of inlets produces the effect of a decrease in the stagnation point pressure. The wall shear stress is 0 at the stagnation point in all 3 cases, but again, an increase in the number of inlets produce a decrease in the maximum wall shear stress. Both static pressure and wall shear stress decays are due to the inter-jet vortices formed.

In the case of equal Reynolds number jets, the interinlet distance influences the stagnation point pressure and wall shear stress. The higher the distance is, the greater the stagnation point pressure (there is less interjet mixing, the jet energy is not lost in vortices formed between jets). Same phenomenon is valid for the wall shear stress, but the difference is not significant.

In the case of 2 jets with different Reynolds numbers, the distribution is no longer symmetric. The inter-inlet distance has no influence on the stagnation point pressure and stagnation point shear stress. When the Reynolds number is smaller, the stagnation point pressure is less than in the case of the greater Reynolds number.

The images acquired during the experiments are consistent with the literature studies: the jet behaves as a free jet after exiting the nozzle, starts mixing with the surrounding fluid and deflects radially. A vortex is being formed, and the flow becomes turbulent - fact underlined by the jet diffusing into air. In the case of several inlets, inter-jet vortices are being formed on each side of the stagnation points. The numerical investigation conducted in ANSYS Fluent in the context of RANS turbulence modelling shows the same flow characteristics as the experimental one.

This work was partially supported by a grant of the Romanian National Authority for Scientific Research, CNCS, UEFISCDI, PHANTOM - Passive flow control for heat and mass transfer enhancement of impinging jets PN-III-P4-ID-PCE-2016-0758, and by a grant of the Romanian space agency ROSA, QUEST - Advanced air diffusion system of the crew quarters for the ISS and deep space habitation systems, STAR-CDI-C3-2016577.

\section{References}

1. P. Hrycak, S. Jachna, D.T. Lee, Letters in Heat and Mass Transfer 1, 63-71 (1974)

2. M.R.S.C. Sastry, B.V.S.S.S. Prasad, A.V.S.S.K.S. Gupta, Indian J. Eng. Mat. Sci. 22, 631-640 (2015)

3. I. Tani, Y. Komatsu, Applied Mechanics, 672-676, Springer (1966)

4. J.W. Gauntner, P. Hrycak, J.N.B. Livingood, Survey of Literature of Flow Characteristics of a Single Turbulent Jet Impinging on a Flat Plate, NASA Technical Note D-5652 (1970)

5. M. Shademan, R. Barron, R. Balachandar, Int. J. Surf. Eng. Mater. Technol. 3, 23-28 (2013)

6. H. Arabnejad, A. Mansoiuri, S.A. Shirazi, B. McLaury, Proc. of the ASME $20144^{\text {th }}$ Joint USEuropean Fluids Engineering Division Summer Meeting FEDSM, 1-6 (2014)

7. C.V. Tu, D.H. Wood, Exp. Therm. Fl. Sci. 13, 364373 (1996)

8. R. van Hout, V. Rinsky, Y.G. Grobman, Int. J. Heat Fluid Fl. 70, 41-58 (2018) 